\title{
Entanglement Robustness in Trace Decreasing Quantum Dynamics
}

\author{
Sergey N. Filippov
}

Steklov Mathematical Institute of Russian Academy of Sciences, Gubkina St. 8, Moscow 119991, Russia

Valiev Institute of Physics and Technology of Russian Academy of Sciences, Nakhimovskii Pr. 34, Moscow 117218, Russia

Moscow Institute of Physics and Technology, Institutskii Per. 9, Dolgoprudny, Moscow Region 141700, Russia

E-mail: sergey.filippov@phystech.edu

Editors: Vinayak Jagadish \& Danko D. Georgiev

Article history: Submitted on September 1, 2021; Accepted on September 6, 2021; Published on September 7, 2021.

ra

race decreasing dynamical maps are as physical as trace preserving ones; however, they are much less studied. Here we overview how the quantum Sinkhorn theorem can be successfully applied to find a two-qubit entangled state which has the strongest robustness against local noises and losses of quantum information carriers. We solve a practically relevant problem of finding an optimal initial encoding to distribute entangled polarized qubits through communication lines with polarization dependent losses and extra depolarizing noise. The longest entanglement lifetime is shown to be attainable with a state that is not maximally entangled.

Quanta 2021; 10: 15-21.

\section{Introduction}

General physical transformations of quantum states are usually associated with quantum channels, i.e., completely positive trace preserving maps (see, e.g., [1-4]). However, if we consider a generally nonprojective quan-

(c) (1) This is an open access article distributed under the terms of the Creative Commons Attribution License CC-BY-3.0, which permits unrestricted use, distribution, and reproduction in any medium, provided the original author and source are credited. tum measurement, then the induced state transformation is a quantum operation, i.e., a completely positive and trace nonincreasing map [5,6].

A mathematical condition of complete positivity is equivalent to a physical condition of positive semidefiniteness for a composite-system density operator, provided the transformation has affected a part of the composite system. The complete positivity condition is long known to be equivalent to positive semidefiniteness of the so-called Choi matrix [7] (see also [8,9]); however, the same matrix was introduced as a dynamical matrix in an earlier publication by Sudarshan, Mathews, and Rau [10], where they implicitly imposed the stronger condition of complete positivity [10, Eq. (16)] instead of the weaker condition of positivity [10, Eq. (12')]. The trace preservation condition takes the form of a matrix equality involving the dynamical matrix [10, Eq. (17)], so the trace-nonincreasing condition takes the form of a matrix inequality.

Note that a mapping from the space of measurement outcomes to the set of quantum operations is known in the literature as a quantum instrument [3, 5, 6]. Repeated applications of the same quantum operation can be used to simulate non-Hermitian quantum dynamics [11,12], whereas repeated applications of the same quantum instrument enable quantum state tomography [13]. Sequentially intervening open system dynamics with quantum 
instruments as in an experiment in Ref. [14] makes it possible to fully learn a generally non-Markovian quantum process [15].

On the other hand, a loss of quantum information carriers in a quantum communication line can be viewed as a quantum operation $\Lambda$ too. In this case, the detection probability $\operatorname{tr}[\Lambda[\varrho]]$ is the probability to successfully implement a quantum operation $\Lambda$ for a given input density operator $\varrho$. If the detection probability is the same for all initial density operators $\varrho$, then $\Lambda$ is merely an attenuated quantum channel, i.e., there exists $0 \leq p \leq 1$ and a quantum channel $\Phi$ such that $\Lambda=p \Phi$. However, quantum physics is much richer and there exist such biased quantum operations $\Lambda$ that $\operatorname{tr}[\Lambda[\varrho]] \neq \operatorname{tr}\left[\Lambda\left[\varrho^{\prime}\right]\right]$ for at least two density operators $\varrho$ and $\varrho^{\prime}[16]$. A prominent example of the latter case is polarization dependent losses [16]17], for which horizontally and vertically polarized photons have different loss probabilities. That asymmetry significantly affects the way one should encode quantum information to reliably transmit quantum information through such a lossy channel, namely, special codes with entangled states are shown to perform better than codes with disentangled states [16].

A one-parameter family of quantum operations $\{\Lambda(t)\}_{t \geq 0}$ represents a process of physical evolution in time $t \geq 0$. If $\varrho(0)$ is an initial density operator of the system, then $\Lambda(t)[\varrho(0)]$ is a subnormalized density operator at time $t$. In a general physical process, the detection probability $\operatorname{tr}[\Lambda(t)[\varrho(0)]]$ does not have to decrease monotonically as quantum information carriers can potentially return to the communication line [18, 19]. Experimentalists usually postselect successful realizations (e.g., in biphoton production [20]) and study dynamics of the conditional output states

$$
\widetilde{\varrho}(t):=\frac{\Lambda(t)[\varrho(0)]}{\operatorname{tr}[\Lambda(t)[\varrho(0)]]} .
$$

Note that the map $\varrho(0) \rightarrow \widetilde{\varrho}(t)$ in nonlinear, so focusing solely on the dynamics of $\widetilde{\varrho}(t)$ may lead to a misidentification of non-Markovianity of $\Lambda(t)[21]$.

We consider a physically relevant problem of two-qubit entanglement distribution through lossy communication lines [22,23]. Let $\varrho_{12}(0)$ be an initial density operator and $\Lambda(t)=\Lambda_{1}(t) \otimes \Lambda_{2}(t)$, where $\Lambda_{i}(t)$ is a qubit operation describing loss and noise accumulated in the $i$-th communication line by time $t$. The goal of this paper is to present a recipe on how one should prepare an initial entangled state $\varrho_{12}(0)$ so that $\widetilde{\varrho}_{12}(t)$ remains entangled during the trace decreasing dynamics for the longest possible time. Entanglement represents a useful resource for quantum communication and device-independent key distribution [24], so its preservation is of high importance to applications. In this paper, we are interested in the fundamental separation between entangled and disentangled states and pay no attention to the "amount" of entanglement. We do that because an arbitrary little but nonzero two-qubit entanglement can potentially be purified: many copies of poorly entangled states can be transformed into a smaller number of almost maximally entangled two-qubit states [25]. In view of this, we focus on the maximum permissible noise level, exceeding which no entanglement-enabled protocol is feasible and below which any entanglement-enabled protocol is fundamentally possible. An entanglement lifetime (a disentangling time, an entanglement sudden death time [26]) of an initial state $\varrho_{12}(0)$ is defined as the minimal time $\tau$ such that $\widetilde{\varrho}_{12}(t)$ is disentangled for all $t \geq \tau$. The maximal possible entanglement lifetime

$$
\widetilde{\tau}=\max _{\varrho_{12}(0)} \tau
$$

provides the fundamental restriction on the length of quantum communications lines for entanglement distribution.

Our study follows a similar analysis made for trace preserving maps [27, 28]; however, the trace decreasing nature of $\Lambda(t)$ modifies the result. If $\Lambda(t)$ is biased, then some states have higher detection probability than others, which increases their contribution to Eq. (1). The optimal state $\varrho_{12}^{\text {opt }}(0)$ makes allowance for that effect and is not maximally entangled in general. A technique to solve the optimization problem posed is based on the quantum Sinkhorn theorem [29-32] that also finds applications in the study of quantum channel capacity [33]. The quantum Sinkhorn theorem relates strictly positive quantum maps with unital ones and enables us to use the known results on entanglement robustness against unital quantum noises [27, 28]. We implement that research programme in Section 2

In addition to a general result, in Section 3, we elaborate the case of polarization dependent losses accompanied by depolarization. This model of loss and noise describes effects in some optical fibers and attracts increasing attention in the literature [34,35]. In this model, each $\Lambda_{i}(t)$ is defined by three parameters: the depolarization rate $\gamma$ and the attenuation coefficients for horizontally and vertically polarized photons, $\gamma_{H}$ and $\gamma_{V}$. We develop a technique on how to find the optimal state $\varrho_{12}^{\text {opt }}(0)$ and the maximal entanglement lifetime provided the above parameters are known for both lines $\Lambda_{1}(t)$ and $\Lambda_{2}(t)$.

\section{Entanglement dynamics and quantum Sinkhorn theorem}

A bipartite density operator $\varrho_{12}$ on a finite dimensional Hilbert space $\mathcal{H}_{1} \otimes \mathcal{H}_{2}$ is called disentangled with respect 
to the bipartition $1 \mid 2$ if it adopts a convex sum representation of the form [36, 37]

$$
\varrho_{12}=\sum_{k} p_{k} \varrho_{1}^{(k)} \otimes \varrho_{2}^{(k)},
$$

where $\left\{p_{k}\right\}_{k}$ is a probability distribution and $\left\{\varrho_{i}^{(k)}\right\}_{k}$ is a collection of density operators on $\mathcal{H}_{i}, i=1,2$. A subnormalized density operator $\varrho_{12}$ with $\operatorname{tr}\left[\varrho_{12}\right] \leq 1$ is disentangled if Eq. (3) represents a conic sum, i.e., $p_{k} \geq 0$ for all $k$.

Let $\{|0\rangle,|1\rangle\}$ be a standard qubit basis such that $\sigma_{x}=$ $|0\rangle\langle 1|+| 1\rangle\left\langle 0\left|, \sigma_{y}=-i\right| 0\right\rangle\langle 1|+i| 1\rangle\langle 0|$, and $\sigma_{z}=|0\rangle\langle 0|-$ $|1\rangle\langle 1|$ are the conventional Pauli operators. The maximally entangled two-qubit state reads $\left|\psi_{+}\right\rangle\left\langle\psi_{+}\right|$, where $\left|\psi_{+}\right\rangle=$ $\frac{1}{\sqrt{2}}(|00\rangle+|11\rangle)$. Note that a reduced density operator of either qubit from the maximally entangled two-qubit pair is in the maximally mixed state $\frac{1}{2} I$, where $I=|0\rangle\langle 0|+$ $|1\rangle\langle 1|$ is the identity operator.

Let us consider such qubit transformations for which the maximally mixed state $\frac{1}{2} I$ is a fixed point. A linear qubit map $\Upsilon$ is called unital if $\Upsilon[I]=I$. If $\Upsilon$ is a unital quantum channel, then the von Neumann entropy of the output state $\Upsilon[\varrho]$ is not less than the von Neumann entropy of the input state $\varrho$ for any density operator $\varrho$.

Two-qubit entanglement dynamics in presence of identical local unital noises of the form $\Upsilon \otimes \Upsilon$ is studied in Ref. [27]. The maximally entangled state $\left|\psi_{+}\right\rangle\left\langle\psi_{+}\right|$is the most robust against local unital noises in the sense that $\Upsilon \otimes \Upsilon\left[\varrho_{12}\right]$ is disentangled for any density operator $\varrho_{12}$ whenever $\Upsilon \otimes \Upsilon\left[\left|\psi_{+}\right\rangle\left\langle\psi_{+}\right|\right]$is disentangled [27].

A generalization of that result for different unital local noises of the form $\Upsilon \otimes \Upsilon^{\prime}$ is obtained in Ref. [28], where a state with the ultimate entanglement robustness is shown to be the maximally entangled state of the form

$$
\left|\psi_{\Upsilon \otimes} \Upsilon^{\prime}\right\rangle=\frac{1}{\sqrt{2}}\left(|\varphi\rangle \otimes|\chi\rangle+\left|\varphi_{\perp}\right\rangle \otimes\left|\chi_{\perp}\right\rangle\right),
$$

with $\left\{|\varphi\rangle,\left|\varphi_{\perp}\right\rangle\right\}\left(\left\{|\chi\rangle,\left|\chi_{\perp}\right\rangle\right\}\right)$ being orthogonal eigenvectors of some traceless eigenoperator of $\Upsilon\left(\Upsilon^{\prime}\right)$.

If both $\Upsilon$ and $\Upsilon^{\prime}$ are diagonal in the basis of Pauli operators $\sigma_{x}, \sigma_{y}, \sigma_{z}$, i.e.,

$$
\begin{aligned}
& \Upsilon[\varrho]=\frac{1}{2} \operatorname{tr}[\varrho] I+\frac{1}{2} \sum_{k=x, y, z} \lambda_{k} \operatorname{tr}\left[\sigma_{k} \varrho\right] \sigma_{k}, \\
& \Upsilon^{\prime}[\varrho]=\frac{1}{2} \operatorname{tr}[\varrho] I+\frac{1}{2} \sum_{k=x, y, z} \lambda_{k}^{\prime} \operatorname{tr}\left[\sigma_{k} \varrho\right] \sigma_{k},
\end{aligned}
$$

and additionally $\lambda_{x} \geq \lambda_{y} \geq \lambda_{z} \geq 0, \lambda_{x}^{\prime} \geq \lambda_{y}^{\prime} \geq \lambda_{z}^{\prime} \geq 0$, then $\left|\psi_{\Upsilon \otimes} \Upsilon^{\prime}\right\rangle=\left|\psi_{+}\right\rangle$.

If parameters $\lambda_{k}$ and $\lambda_{k}^{\prime}$ in Eqs. (5) and (6) are functions of time, then we deal with a local unital dynamics $\Upsilon(t) \otimes$
$\Upsilon^{\prime}(t)$ which preserves entanglement of the maximally entangled state as long as [28]

$$
\lambda_{x}(t) \lambda_{x}^{\prime}(t)+\lambda_{y}(t) \lambda_{y}^{\prime}(t)+\lambda_{z}(t) \lambda_{z}^{\prime}(t)>1 .
$$

A linear map $\Lambda$ on operators in $\mathcal{H}$ is called strictly positive if $\Lambda[\varrho]$ is positive definite for all nonzero positive semidefinite operators $\varrho$. Strictly positive maps belong to the interior of the cone of positivity preserving maps [31] and are also referred to as positivity improving ones [30]. If $\Lambda$ is strictly positive, then by Proposition 2.32 in [32] there exist positive definite operators $A$ and $B$ such that the map

$$
\Upsilon=\Phi_{A} \circ \Lambda \circ \Phi_{B}
$$

is trace preserving and unital. Here, $\Phi_{X}[\varrho]=X \varrho X^{\dagger}$ and $\circ$ denotes the map concatenation. If $\Lambda$ is completely positive and strictly positive, then $\Upsilon$ is a unital quantum channel. The relation (8) is known as the quantum Sinkhorn theorem originally discovered in Ref. [29], rediscovered for positivity improving completely positive maps in Ref. [30], and finally clarified in Refs. [31,32]. One can express the operators $A$ and $B$ through $A=\sqrt{S}$ and $B=\left(\Lambda^{\dagger}[S]\right)^{-1 / 2}$, where a positive definite operator $S$ is a fixed point of the map $F[S]=\left(\Lambda\left[\left(\Lambda^{\dagger}[S]\right)^{-1}\right]\right)^{-1}$, where $\Lambda^{\dagger}$ denotes a dual linear map with respect to $\Lambda$, i.e., $\operatorname{tr}\left[\Lambda^{\dagger}[X] Y\right]=\operatorname{tr}[X \Lambda[Y]]$ for all $X, Y$. A methodology to explicitly find operators $A$ and $B$ for trace preserving qubit maps $\Lambda$ is given in Ref. [28]; however, a methodology for trace decreasing maps is still missing. In Section 3 , we partially fill this gap for a physically relevant scenario of combined noise and loss.

The inverse relation to Eq. (8) reads

$$
\Lambda=\Phi_{A^{-1}} \circ \Upsilon \circ \Phi_{B^{-1}}
$$

and enables us to find the structure of the optimal state $\varrho^{\text {opt}}(0)$. Indeed, since the map $\Phi_{X}$ has a single Kraus operator $X$, the operator $\Lambda \otimes \Lambda^{\prime}[\varrho(0)]$ is entangled if and only if $\left(\Upsilon \otimes \Upsilon^{\prime}\right) \circ\left(\Phi_{B^{-1}} \otimes \Phi_{B^{\prime-1}}\right)[\varrho(0)]$ is entangled. On the other hand, the most robust entangled state against the noise $\Upsilon \otimes \Upsilon^{\prime}$ is $\left|\psi_{\Upsilon \otimes \Upsilon^{\prime}}\right\rangle\left\langle\psi_{\Upsilon \otimes} \Upsilon^{\prime}\right|$ given by Eq. (4), so $\left(\Phi_{B^{-1}} \otimes \Phi_{B^{\prime-1}}\right)[\varrho(0)] \propto\left|\psi \Upsilon \otimes \Upsilon^{\prime}\right\rangle\left\langle\psi_{\Upsilon \otimes} \Upsilon^{\prime}\right|$. Inverting the map $\Phi_{B^{-1}} \otimes \Phi_{B^{\prime-1}}$, we get $\varrho^{\text {opt }}(0)=\left|\psi_{\Lambda \otimes \Lambda^{\prime}}\right\rangle\left\langle\psi_{\Lambda \otimes \Lambda^{\prime}}\right|$, where

$$
\left|\psi_{\Lambda \otimes \Lambda^{\prime}}\right\rangle=\frac{B(\widetilde{\tau}) \otimes B^{\prime}(\widetilde{\tau})\left|\psi \Upsilon \otimes \Upsilon^{\prime}\right\rangle}{\sqrt{\left\langle\psi \Upsilon \otimes \Upsilon^{\prime}\left|B^{\dagger}(\widetilde{\tau}) B(\widetilde{\tau}) \otimes B^{\prime}(\widetilde{\tau})^{\dagger} B^{\prime}(\widetilde{\tau})\right| \psi \Upsilon \otimes \Upsilon^{\prime}\right\rangle}}
$$

and $\widetilde{\tau}$ is the maximal entanglement lifetime under noise $\Upsilon(t) \otimes \Upsilon^{\prime}(t)$ determined in [28, Proposition 1]. In the case when both $\lambda_{x}(t) \geq \lambda_{y}(t) \geq \lambda_{z}(t) \geq 0$ and $\lambda_{x}^{\prime}(t) \geq \lambda_{y}^{\prime}(t) \geq$ $\lambda_{z}^{\prime}(t) \geq 0$, one can substitute $\left|\psi_{+}\right\rangle$for $\left|\psi_{\Upsilon \otimes} \Upsilon^{\prime}\right\rangle$ and find the maximal entanglement lifetime $\widetilde{\tau}$ as the smallest $t>0$ for which the inequality (7) is violated. 


\section{Entanglement distribution in presence of depolarization and polarization dependent losses}

The effect of polarization dependent losses is that the states with different polarization are attenuated differently [16, 17, 34, 35]. Let the horizontally and vertically polarized states be those that are the least and most attenuated, or vice versa. By $\gamma_{H}$ and $\gamma_{V}$ denote the attenuation rates for horizontally and vertically polarized photons, respectively. Let $(|H\rangle,|V\rangle)$ be a conventional basis composed of the horizontally and vertically polarized one-photon states. In what follows, we will identify the basis $(|H\rangle,|V\rangle)$ with the standard basis $(|0\rangle,|1\rangle)$ used in Section 2. Then the combined effect of polarization dependent losses and depolarization with the rate $\gamma$ on a polarization qubit is described the following master equation:

$$
\begin{aligned}
\frac{d \varrho(t)}{d t}= & -\frac{1}{2}\left\{\gamma_{H}|H\rangle\left\langle H\left|+\gamma_{V}\right| V\right\rangle\langle V|, \varrho(t)\right\} \\
& +\frac{\gamma}{4} \sum_{k=x, y, z}\left(\sigma_{k} \varrho(t) \sigma_{k}-\varrho(t)\right),
\end{aligned}
$$

where $\{\cdot, \cdot\}$ stands for the anticommutator. Eq. (11) defines the dynamical semigroup $\Lambda(t)$ that is trace decreasing if $\gamma_{H}>0$ and $\gamma_{V}>0$.

Let us consider a matrix representation (see, e.g., [6]) of the qubit map $\Lambda(t)$, i.e., a $4 \times 4$ matrix $M(t)$ whose elements are defined through

$$
M_{i j}(t)=\frac{1}{2} \operatorname{tr}\left[\sigma_{i} \Lambda(t)\left[\sigma_{j}\right]\right], \quad i, j=0, x, y, z,
$$

where $\sigma_{0}=I$. Some tedious yet straightforward algebra yields

$$
\begin{aligned}
& M(t)=\left(\begin{array}{cccc}
a(t) & 0 & 0 & b(t) \\
0 & c(t) & 0 & 0 \\
0 & 0 & c(t) & 0 \\
b(t) & 0 & 0 & d(t)
\end{array}\right), \\
& a(t)=e^{-\frac{1}{2}\left(\gamma+\gamma_{H}+\gamma_{V}\right) t}\left(\cosh \frac{\sqrt{\gamma^{2}+\left(\gamma_{H}-\gamma_{V}\right)^{2}} t}{2}+\frac{\gamma}{\sqrt{\gamma^{2}+\left(\gamma_{H}-\gamma_{V}\right)^{2}}} \sinh \frac{\sqrt{\gamma^{2}+\left(\gamma_{H}-\gamma_{V}\right)^{2}} t}{2}\right), \\
& b(t)=-\frac{\gamma_{H}-\gamma_{V}}{\sqrt{\gamma^{2}+\left(\gamma_{H}-\gamma_{V}\right)^{2}}} e^{-\frac{1}{2}\left(\gamma+\gamma_{H}+\gamma_{V}\right) t} \sinh \frac{\sqrt{\gamma^{2}+\left(\gamma_{H}-\gamma_{V}\right)^{2}} t}{2}, \\
& c(t)=e^{-\frac{1}{2}\left(2 \gamma+\gamma_{H}+\gamma_{V}\right) t}, \\
& d(t)=e^{-\frac{1}{2}\left(\gamma+\gamma_{H}+\gamma_{V}\right) t}\left(\cosh \frac{\sqrt{\gamma^{2}+\left(\gamma_{H}-\gamma_{V}\right)^{2}} t}{2}-\frac{\gamma}{\sqrt{\gamma^{2}+\left(\gamma_{H}-\gamma_{V}\right)^{2}}} \sinh \frac{\sqrt{\gamma^{2}+\left(\gamma_{H}-\gamma_{V}\right)^{2}} t}{2}\right) .
\end{aligned}
$$

To apply the quantum Sinkhorn theorem to a map $\Lambda(t)$ with the matrix representation (13) we need to find a fixed point of the map $F[S]$, i.e., to solve a matrix equation

$$
S=\left(\Lambda(t)\left[\left(\Lambda(t)^{\dagger}[S]\right)^{-1}\right]\right)^{-1} .
$$

Note that in our case $\Lambda(t)^{\dagger}=\Lambda(t)$. Due to the phase covariance of $\Lambda(t)$ [38], we seek the operator $S$ in the form of an ansatz $S=I+s \sigma_{z}$. If $b(t) \neq 0$, this results in the following solution:

$$
s(t)=-\frac{a(t)+d(t)-\sqrt{[a(t)+d(t)]^{2}-4 b^{2}(t)}}{2 b(t)} .
$$

Note that $S=I+s \sigma_{z}$ is positive definite if $a(t)+d(t) \geq$ The obtained expressions satisfy the conditions $\lambda_{x}(t) \geq$ $2|b(t)| \neq 0$ which is automatically fulfilled for expressions (14)-(17) if $t>0$. Substituting $A=\sqrt{S}$ and
$B=\left(\Lambda(t)^{\dagger}[S]\right)^{-1 / 2}$ into Eq. $(8)$, we get the corresponding unital map $\Upsilon(t)$ that has the form (5) with parameters

$$
\begin{aligned}
& \lambda_{x}(t)=\lambda_{y}(t)=\frac{2 c(t)}{a(t)-d(t)+\sqrt{[a(t)+d(t)]^{2}-4 b^{2}(t)}}, \\
& \lambda_{z}(t)=\frac{4\left[a(t) d(t)-b^{2}(t)\right]}{\left\{a(t)-d(t)+\sqrt{[a(t)+d(t)]^{2}-4 b^{2}(t)}\right\}^{2}} \cdot
\end{aligned}
$$
end of Section 2 . 


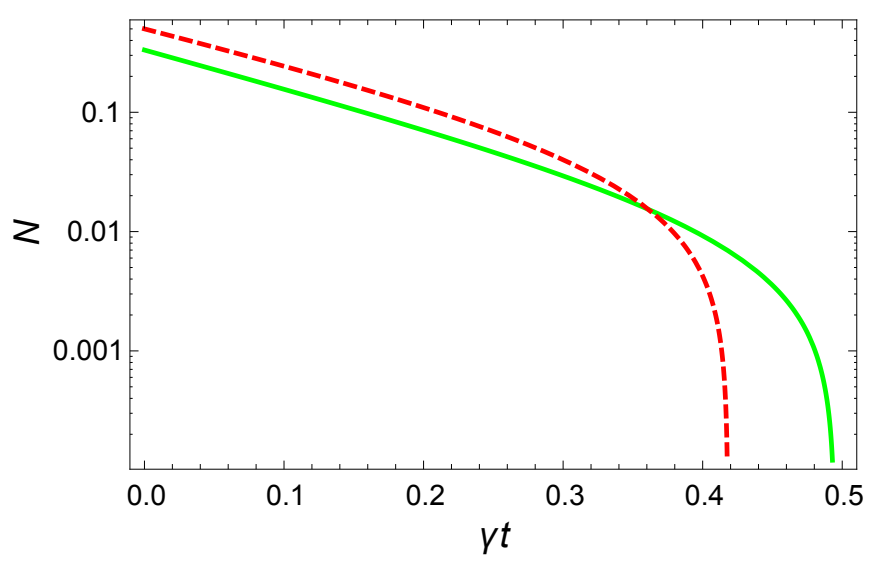

Figure 1: Effect of local depolarization and polarization dependent losses, $\Lambda(t) \otimes \Lambda(t)$ with $\gamma_{H}=\gamma$ and $\gamma_{V}=5 \gamma$, on two-qubit entanglement dynamics (negativity) for different initial states: the maximally entangled one (red dashed line) and the optimal one (green solid line).

Given two communication lines $\Lambda(t)$ and $\Lambda^{\prime}(t)$ with parameters $\gamma_{H}, \gamma_{V}, \gamma$ and $\gamma_{H}^{\prime}, \gamma_{V}^{\prime}, \gamma^{\prime}$, respectively, the maximal entanglement lifetime $\widetilde{\tau}$ is a solution of the equation

$$
\lambda_{x}(\widetilde{\tau}) \lambda_{x}^{\prime}(\widetilde{\tau})+\lambda_{y}(\widetilde{\tau}) \lambda_{y}^{\prime}(\widetilde{\tau})+\lambda_{z}(\widetilde{\tau}) \lambda_{z}^{\prime}(\widetilde{\tau})=1 .
$$

The optimal state $\varrho^{\text {opt }}(0)=\left|\psi_{\Lambda \otimes \Lambda^{\prime}}\right\rangle\left\langle\psi_{\Lambda \otimes \Lambda^{\prime}}\right|$ is defined by the normalized vector

$$
\left|\psi_{\Lambda \otimes \Lambda^{\prime}}\right\rangle \propto B(\widetilde{\tau}) \otimes B^{\prime}(\widetilde{\tau})(|H H\rangle+|V V\rangle),
$$

where

$$
\begin{aligned}
B(\widetilde{\tau})= & \frac{|H\rangle\langle H|}{\sqrt{a(\widetilde{\tau})+b(\widetilde{\tau})+s(\widetilde{\tau})[b(\widetilde{\tau})+d(\widetilde{\tau})]}} \\
& +\frac{|V\rangle\langle V|}{\sqrt{a(\widetilde{\tau})-b(\widetilde{\tau})+s(\widetilde{\tau})[b(\widetilde{\tau})-d(\widetilde{\tau})]}}
\end{aligned}
$$

and $B^{\prime}(\widetilde{\tau})$ is obtained from Eq. (24) by replacing $a \rightarrow a^{\prime}$, $b \rightarrow b^{\prime}, c \rightarrow c^{\prime}, d \rightarrow d^{\prime}$.

Despite the fact that the final expression for the optimal state (23) is rather involved, it is analytically derived and can be further explored. In Fig. 1 we depict the evolution of the entanglement quantifier $N$ called negativity [37,39] for the maximally entangled initial state $\left|\psi_{+}\right\rangle\left\langle\psi_{+}\right|$and the optimal state (23). If $\varrho$ is a density operator of a bipartite system, then $N(\varrho)=\frac{1}{2}\left(\|(\operatorname{Id} \otimes T)[\varrho]\|_{1}-1\right)$, where Id is the identity transformation, $T$ is the transposition in the standard basis, and $\|X\|_{1}=\operatorname{tr} \sqrt{X^{\dagger} X}$. A two qubit state $\varrho$ is entangled if and only if $N(\varrho)>0$ [40]. Fig. 1] shows that although the state $\Lambda(t) \otimes \Lambda^{\prime}(t)\left[\varrho^{\mathrm{opt}}(0)\right]$ exhibits less entanglement in the beginning of evolution as compared to $\Lambda(t) \otimes \Lambda^{\prime}(t)\left[\left|\psi_{+}\right\rangle\left\langle\psi_{+}\right|\right]$, the inverse relation takes place after some time. Finally, after some time $\Lambda(t) \otimes \Lambda^{\prime}(t)\left[\left|\psi_{+}\right\rangle\left\langle\psi_{+}\right|\right]$ becomes separable whereas $\Lambda(t) \otimes \Lambda^{\prime}(t)\left[\varrho^{\text {opt }}(0)\right]$ remains entangled. This phenomenon illustrates how a less entangled optimal state outperforms the maximally entangled state in long term.

\section{Conclusions}

Using a general technique of the quantum Sinkhorn theorem and the previously known results for trace preserving quantum maps, we have studied the two-qubit entanglement lifetime under arbitrary trace decreasing local operations (Section 2). An analytical treatment of the quantum Sinkhorn theorem for a particular quantum map $\Lambda(t)$ is a challenge because Eq. (18) is rather difficult to resolve. Nevertheless, we have managed to derive the explicit form of the Sinkhorn theorem for trace decreasing qubit operations with the matrix representation (13). This analytical result advances our understanding of the quantum Sinkhorn theorem beyond the trace-preserving case of phase-covariant qubit maps, which was the only nontrivial class of maps with explicit analytical expressions for $\Upsilon, A$, and $B$ [28, 33] known before this work.

We applied the proposed approach to the analysis of entanglement dynamics of polarization-encoded two-qubit states subjected to the combined effect of depolarization and polarization dependent losses (Section 3). Our goal was to find the longest entanglement lifetime $\widetilde{\tau}$ among all possible initial states. The longest entanglement lifetime determines a fundamental noise level exceeding which no entanglement-enabled protocol can be implemented. We expressed $\widetilde{\tau}$ as a solution of the analytically derived Eq. (22). The optimal initial state (23) - that exhibits the strongest robustness against depolarization and polarization dependent losses - is not maximally entangled if $\gamma_{H} \neq \gamma_{V}$. The optimal state makes allowance for the difference in attenuation coefficients $\left(\gamma_{H}\right.$ and $\left.\gamma_{V}\right)$ and has a higher contribution of those polarization component, which decays more rapidly.

\section{Acknowledgements}

The study in Sections 2, 3, and 4 was supported by the Russian Science Foundation under Project No. 19-11-00086 and performed in Steklov Mathematical Institute of Russian Academy of Sciences. The review in Section 1 was written in Moscow Institute of Physics and Technology as well as in Valiev Institute of Physics and Technology of Russian Academy of Sciences, where the author was supported by Program No. 0066-2019-0005 of the Russian Ministry of Science and Higher Education. 


\section{References}

[1] M. A. Nielsen, I. L. Chuang. Quantum Computation and Quantum Information. Cambridge University Press, Cambridge, 2010. doi:10.1017/ cbo9780511976667.

[2] H.-P. Breuer, F. Petruccione. The Theory of Open Quantum Systems. Oxford University Press, Oxford, 2007.

[3] A. S. Holevo. Quantum Systems, Channels, Information: A Mathematical Introduction. Vol. 16 of $D e$ Gruyter Studies in Mathematical Physics. Walter de Gruyter, Berlin, 2012.

[4] V. Jagadish, F. Petruccione. An invitation to quantum channels. Quanta 2018; 7:54-67. doi:10. 12743/quanta.v7i1.77.

[5] E. B. Davies, J. T. Lewis. An operational approach to quantum probability. Communications in Mathematical Physics 1970; 17(3):239-260. doi:10. $1007 / \mathrm{bf01647093.}$

[6] T. Heinosaari, M. Ziman. The Mathematical Language of Quantum Theory: From Uncertainty to Entanglement. Cambridge University Press, Cambridge, 2012. doi : 10.1017/cbo9781139031103.

[7] M.-D. Choi. Completely positive linear maps on complex matrices. Linear Algebra and its Applications 1975; 10(3):285-290. doi:10.1016/00243795(75) 90075-0.

[8] J. de Pillis. Linear transformations which preserve Hermitian and positive semidefinite operators. $\mathrm{Pa}$ cific Journal of Mathematics 1967; 23(1):129-137. doi : 10.2140/pjm.1967.23.129.

[9] A. Jamiołkowski. Linear transformations which preserve trace and positive semidefiniteness of operators. Reports on Mathematical Physics 1972; 3(4):275-278. doi:10.1016/0034-4877(72) 90011-0.

[10] E. C. G. Sudarshan, P. M. Mathews, J. Rau. Stochastic dynamics of quantum-mechanical systems. Physical Review 1961; 121(3):920-924. doi:10.1103/ PhysRev.121.920.

[11] I. A. Luchnikov, S. N. Filippov. Quantum evolution in the stroboscopic limit of repeated measurements. Physical Review A 2017; 95(2):022113. doi:10. 1103/PhysRevA.95.022113
[12] R. Grimaudo, A. Messina, A. Sergi, N. V. Vitanov, S. N. Filippov. Two-qubit entanglement generation through non-Hermitian Hamiltonians induced by repeated measurements on an ancilla. Entropy 2020; 22(10):1184. doi : 10.3390/e22101184.

[13] V. A. Zhuravlev, S. N. Filippov. Quantum state tomography via sequential uses of the same informationally incomplete measuring apparatus. Lobachevskii Journal of Mathematics 2020; 41(12):2405-2414. doi:10.1134/ s1995080220120434.

[14] L. Xiang, Z. Zong, Z. Zhan, Y. Fei, C. Run, Y. Wu, W. Jin, Z. Jia, P. Duan, J. Wu, Y. Yin, G. Guo. Quantify the non-Markovian process with intervening projections in a superconducting processor 2021; arXiv:2105.03333.

[15] I. A. Luchnikov, S. V. Vintskevich, D. A. Grigoriev, S. N. Filippov. Machine learning non-Markovian quantum dynamics. Physical Review Letters 2020; 124(14):140502. doi:10.1103/PhysRevLett. 124.140502 .

[16] S. N. Filippov. Capacity of trace decreasing quantum operations and superadditivity of coherent information for a generalized erasure channel. Journal of Physics A: Mathematical and Theoretical 2021; 54(25):255301. doi : 10 . 1088/1751-8121/ abfd61.

[17] N. Gisin, B. Huttner. Combined effects of polarization mode dispersion and polarization dependent losses in optical fibers. Optics Communications 1997; 142(1):119-125. doi:10.1016/S00304018(97)00236-8.

[18] J. Jin, V. Giovannetti, R. Fazio, F. Sciarrino, P. Mataloni, A. Crespi, R. Osellame. All-optical nonMarkovian stroboscopic quantum simulator. Physical Review A 2015; 91(1):012122. doi : 10.1103/ PhysRevA.91.012122.

[19] A. Cuevas, A. Geraldi, C. Liorni, L. D. Bonavena, A. De Pasquale, F. Sciarrino, V. Giovannetti, P. Mataloni. All-optical implementation of collision-based evolutions of open quantum systems. Scientific Reports 2019; 9(1):3205. doi:10.1038/s41598019-39832-9.

[20] E. V. Moreva, G. A. Maslennikov, S. S. Straupe, S. P. Kulik. Realization of four-level qudits using biphotons. Physical Review Letters 2006; 97(2):023602. doi:10.1103/PhysRevLett.97.023602. 
[21] S. N. Filippov. Trace decreasing quantum dynamical maps: divisibility and entanglement dynamics 2021; arXiv:2108.13372

[22] S. N. Filippov. Quantum mappings and characterization of entangled quantum states. Journal of Mathematical Sciences 2019; 241(2):210-236. doi : 10.1007/s10958-019-04418-3.

[23] S. N. Filippov. Tensor products of quantum mappings. Journal of Mathematical Sciences 2021; 252(1):116-124. doi:10.1007/s10958-02005146-9.

[24] F. Xu, B. Qi, Z. Liao, H.-K. Lo. Long distance measurement-device-independent quantum key distribution with entangled photon sources. Applied Physics Letters 2013; 103(6):061101. doi:10. $1063 / 1.4817672$

[25] M. Horodecki, P. Horodecki, R. Horodecki. Inseparable two spin- $\frac{1}{2}$ density matrices can be distilled to a singlet form. Physical Review Letters 1997; 78(4):574-577. doi : 10.1103/PhysRevLett. 78. 574 .

[26] M. P. Almeida, F. de Melo, M. Hor-Meyll, A. Salles, S. P. Walborn, P. H. Souto Ribeiro, L. Davidovich. Environment-induced sudden death of entanglement. Science 2007; 316(5824):579-582. doi : 10.1126/ science.1139892

[27] S. N. Filippov, T. Rybár, M. Ziman. Local twoqubit entanglement-annihilating channels. Physical Review A 2012; 85(1):012303. doi:10.1103/ PhysRevA.85.012303.

[28] S. N. Filippov, V. V. Frizen, D. V. Kolobova. Ultimate entanglement robustness of two-qubit states against general local noises. Physical Review A 2018; 97(1):012322. doi:10.1103/PhysRevA. 97.012322 .

[29] L. Gurvits. Classical complexity and quantum entanglement. Journal of Computer and System Sciences 2004; 69(3):448-484. doi:10.1016/j. jcss.2004.06.003.

[30] T. T. Georgiou, M. Pavon. Positive contraction mappings for classical and quantum Schrödinger systems. Journal of Mathematical Physics 2015; 56(3):033301. doi : 10.1063/1.4915289.

[31] G. Aubrun, S. J. Szarek. Two proofs of Størmer's theorem 2015; arXiv: 1512.03293.
[32] G. Aubrun, S. J. Szarek. Alice and Bob Meet Banach: The Interface of Asymptotic Geometric Analysis and Quantum Information Theory. Vol. 223 of Mathematical Surveys and Monographs. American Mathematical Society, Providence, Rhode Island, 2017. doi : 10. $1090 /$ surv/223.

[33] S. N. Filippov. Lower and upper bounds on nonunital qubit channel capacities. Reports on Mathematical Physics 2018; 82(2):149-159. doi:10.1016/ S0034-4877 (18)30083-1.

[34] B. T. Kirby, D. E. Jones, M. Brodsky. Effect of polarization dependent loss on the quality of transmitted polarization entanglement. Journal of Lightwave Technology 2019; 37(1):95-102. doi:10.1109/ jlt.2018.2879754.

[35] C. Li, M. Curty, F. Xu, O. Bedroya, H.-K. Lo. Secure quantum communication in the presence of phase- and polarization-dependent loss. Physical Review A 2018; 98(4):042324. doi : 10.1103/ PhysRevA.98.042324.

[36] R. F. Werner. Quantum states with Einstein-Podolsky-Rosen correlations admitting a hidden-variable model. Physical Review A 1989; 40(8):4277-4281. doi:10.1103/PhysRevA.40.4277.

[37] R. Horodecki, P. Horodecki, M. Horodecki, K. Horodecki. Quantum entanglement. Reviews of Modern Physics 2009; 81(2):865942. arXiv:quant-ph/0702225. doi: 10.1103/RevModPhys.81.865.

[38] S. N. Filippov, A. N. Glinov, L. Leppäjärvi. Phase covariant qubit dynamics and divisibility. Lobachevskii Journal of Mathematics 2020; 41(4):617-630. doi: 10.1134/s1995080220040095.

[39] K. Życzkowski, P. Horodecki, A. Sanpera, M. Lewenstein. Volume of the set of separable states. Physical Review A 1998; 58(2):883-892. doi : 10.1103/PhysRevA. 58.883.

[40] M. Horodecki, P. Horodecki, R. Horodecki. Separability of mixed states: necessary and sufficient conditions. Physics Letters A 1996; 223(1):18. arXiv:quant-ph/9605038 doi:10.1016/ S0375-9601(96)00706-2. 